Pacific Journal of Mathematics

GENERATORS OF POSITIVE $C_{0}$-SEMIGROUPS 


\title{
GENERATORS OF POSITIVE $C_{0}$-SEMIGROUPS
}

\author{
Shizuo MiYajima AND Noboru OKazawa
}

By the use of abstract Kato's inequality for generators of positive $C_{0}$-semigroups, it is shown that a differential operator with smooth coefficients (with natural domain) is of order at most 2 and (degenerate) elliptic provided it generates a positive $C_{0}$-semigroup on $L^{p}\left(\mathbf{R}^{n}\right)(1 \leq p$ $<\infty$ ). Conversely it is also shown that a certain second order elliptic differential operator with a singular 0 th order coefficient generates a positive $C_{0}$-semigroup on $L^{p}\left(\mathbf{R}^{n}\right)(1<p<\infty)$.

1. Introduction. It has long been known that a certain second order elliptic differential operator generates a positive ( $=$ positive-cone preserving) $C_{0}$-semigroup on various spaces. As a recent result in this direction, we mention Baoswan Wong-Dzung [16], in which it is proved by a functional analytic method that an possibly degenerate elliptic second order differential operator generates a positive $C_{0}$-semigroup on $L^{p}\left(\mathbf{R}^{n}\right)$ $(1<p<\infty)$. One of the purpose of this paper is to show that, conversely, if a differential operator generates a positive $C_{0}$-semigroup on $L^{p}\left(\mathbf{R}^{n}\right)$, then it must be (degenerate) elliptic and of order at most 2 . This will be shown in $\S 3$ by using abstract Kato's inequality recently established for generators of positive $C_{0}$-semigroups [1]. On the contrary, we show in $\S 4$ that a certain second order elliptic differential operator with a singular 0th order coefficient generates a positive $C_{0}$-semigroup on $L^{p}\left(\mathbf{R}^{n}\right)(1<p<$ $\infty)$. This will be proved by applying a perturbation theory obtained by one of the authors to the result of [16]. Necessary definitions and expositions are given in $\$ 2$. In this paper we exclusively work with real Banach lattices, especially real $L^{p}\left(\mathbf{R}^{n}\right)$, since positive $C_{0}$-semigroups and their generators are real operators.

2. Abstract Kato's inequality and dispersive operators. Let $\left\{T_{t}\right\}_{t \geq 0}$ be a $C_{0}$-semigroup of linear operators on a Banach lattice $E$ with generator $A$. Then $\left\{T_{t}\right\}_{t \geq 0}$ is said to be positive if $T_{t}$ is positive for any $t \geq 0$, namely $T_{t} u \geq 0$ whenever $u \geq 0$. The resolvent $(\lambda-A)^{-1}$ of $A$ is denoted by $R(\lambda, A)$. Then the equalities

$$
T_{t}=\operatorname{s-lim}_{n \rightarrow \infty}[(n / t) R((n / t), A)]^{n} \quad(t>0),
$$


and

$$
\left.R(\lambda, A)=\int_{0}^{\infty} e^{-\lambda t} T_{t} d t \quad \text { (for sufficiently large } \lambda\right)
$$

immediately imply that $\left\{T_{t}\right\}_{t \geq 0}$ is positive if and only if $R(\lambda, A)$ is positive for sufficiently large $\lambda$. But in practice the resolvent $R(\lambda, A)$ is rather distant from $A$ itself. So it is desirable to obtain an intrinsic characterization of the generators of positive $C_{0}$-semigroups. As to this problem, Simon [15] remarked that Kato's inequality for the Laplacian $\Delta$ (Kato [5]) is relevant to the positivity of $e^{\Delta t}$ on $L^{2}$. Subsequently Nagel-Uhlig [6] conjectured and partly confirmed that the positivity of a semigroup is characterized by an abstract version of Kato's inequality for the generator.

To formulate Kato's inequality for generators of positive $C_{0}$-semigroups, we need the notion of "signum operators". Let $E$ be a $\sigma$-order complete Banach lattice and let $u \in E$. Then there exists a unique bounded linear operator " $\operatorname{sgn} u$ " on $E$ satisfying

$$
\begin{gathered}
|(\operatorname{sgn} u) v| \leq|v|, \quad v \in E \\
(\operatorname{sgn} u) v=0 \quad \text { if } u \perp v \\
(\operatorname{sgn} u) u=|u|
\end{gathered}
$$

If the band projection (Schaefer [12], p. 61) onto the band generated by $v \in E_{+}$is denoted by $P_{v}$,

$$
\operatorname{sgn} u=P_{u^{+}}-P_{u^{-}} .
$$

For the details of the definition of signum operators, we refer the reader to Nagel-Uhlig [6]. In case $E=L^{p}\left(\mathbf{R}^{n}\right)(1 \leq p \leq \infty), \operatorname{sgn} u$ is the multiplication operator $v \mapsto m v$, where $m \in L^{\infty}\left(\mathbf{R}^{n}\right)$ is defined as follows:

$$
m(x)=\left\{\begin{aligned}
1 & (u(x)>0) \\
-1 & (u(x)<0) \\
0 & (u(x)=0)
\end{aligned}\right.
$$

Next we recall the notion of Yosida approximation of the generator of a $C_{0}$-semigroup. Let $A$ be the generator of a $C_{0}$-semigroup $\left\{T_{t}\right\}_{t \geq 0}$ on a Banach space $X$ with domain $D(A)$. Then $R(\lambda, A):=(\lambda-A)^{-1}$ exists for sufficiently large $\lambda \in \mathbf{R}$, and the operator $A_{\lambda}:=\lambda A R(\lambda, A)=$ $\lambda^{2} R(\lambda, A)-\lambda$ is called the Yosida approximation of $A$. It is well known that $\mathrm{s}-\lim _{\lambda \rightarrow \infty} A_{\lambda} u=A u$ for $u \in D(A)$ and $T_{t} u=\mathrm{s}-\lim _{\lambda \rightarrow \infty} e^{t A_{\lambda}} u$ holds for any $u \in X$ and $t \geq 0$ (Pazy [8], Chap. 1, Theorem 5.5). 
Now we state Proposition 1.1 in Arendt [2] with a different proof.

Proposition 2.1 (Arendt [2]). Let $\left\{T_{t}\right\}_{t \geq 0}$ be a positive $C_{0}$-semigroup on an $\sigma$-order complete Banach lattice $E$ with generator $A$. Then the following abstract Kato's inequality holds:

$$
\forall u \in D(A) \forall f \in D\left(A^{*}\right) \cap E_{+}^{*}\left\langle|u|, A^{*} f\right\rangle \geq\langle(\operatorname{sgn} u) A u, f\rangle,
$$

where $A^{*}$ designates the adjoint of $A$.

Proof. As remarked in the first paragraph of this section, $R(\lambda, A)$ is positive for large $\lambda \in \mathbf{R}$. Hence for such $\lambda$ and $u \in E$,

$$
\begin{aligned}
A_{\lambda}|u| & =\lambda^{2} R(\lambda, A)|u|-\lambda|u| \\
& \geq \lambda^{2}|R(\lambda, A) u|-\lambda|u| \\
& \geq \lambda^{2}(\operatorname{sgn} u) R(\lambda, A) u-\lambda(\operatorname{sgn} u) u \\
& =(\operatorname{sgn} u) A_{\lambda} u .
\end{aligned}
$$

Therefore

$$
\left\langle|u|, A_{\lambda}^{*} f\right\rangle=\left\langle A_{\lambda}|u|, f\right\rangle \geq\left\langle(\operatorname{sgn} u) A_{\lambda} u, f\right\rangle
$$

holds for any $u \in D(A)$ and $f \in D\left(A^{*}\right) \cap E_{+}^{*}$. Letting $\lambda \rightarrow \infty$ we obtain the abstract Kato's inequality since $\operatorname{s}^{*} \lim _{\lambda \rightarrow \infty} A_{\lambda} u=A u$ and $\mathrm{w}^{*}-\lim _{\lambda \rightarrow \infty} A_{\lambda}^{*} f=A^{*} f$.

RemarK 2.2. Arendt ([1], Proposition 1.2) first established abstract Kato's inequality for generators of positive semigroups. His proof is very simple once one admits that $\mathrm{w}^{*}-\lim _{t \downarrow 0}\left(T_{t}^{*} f-f\right) / t=A^{*} f$ for $f \in D\left(A^{*}\right)$. Before the publication of [1], with essentially the same proof as above, the present authors independently obtained a slightly weaker version of Proposition 2.1 which was sufficient to get the result in $\$ 3$ (Theorem 3.6). The authors' result was weak in the sense that $A^{*}$ was replaced by the generator of the dual semigroup of $\left\{T_{t}\right\}_{t \geq 0}$ (Yosida [17], p. 272). See also Schep [13].

The following is an immediate corollary to Proposition 2.1.

Proposition 2.3. Let $E=L^{p}\left(\mathbf{R}^{n}\right) \quad(1 \leq p<\infty, n \in \mathbf{N})$ and let $\left\{T_{t}\right\}_{t \geq 0}$ be a positive $C_{0}$-semigroup on $E$ with generator $A$. Suppose $A$ satisfies $D(A) \cap D\left(A^{*}\right) \supset C_{0}^{\infty}\left(\mathbf{R}^{n}\right)$ and $A$ is given as a differential operator on $C_{0}^{\infty}\left(\mathbf{R}^{n}\right)$ :

$$
A u(x)=\sum_{|\alpha| \leq m} a_{\alpha}(x) D^{\alpha} u(x) \quad\left(u \in C_{0}^{\infty}\left(\mathbf{R}^{n}\right)\right)
$$


where $\alpha=\left(\alpha_{1}, \alpha_{2}, \ldots, \alpha_{n}\right)$ is a multi-index, $|\alpha|=\alpha_{1}+\alpha_{2}+\cdots+\alpha_{n}, D^{\alpha}$ $=\left(\partial / \partial x_{1}\right)^{\alpha_{1}} \cdots\left(\partial / \partial x_{n}\right)^{\alpha_{n}}$, and $a_{\alpha} \in C^{\infty}\left(\mathbf{R}^{n}\right)$.

Then $A$ satisfies the distributional Kato's inequality (see Definition 2.4 below):

$$
\forall u \in C_{0}^{\infty}\left(\mathbf{R}^{n}\right) \quad A|u| \geq(\operatorname{sgn} u) A u
$$

REMARK 2.4. We note that if $C_{0}^{\infty}\left(\mathbf{R}^{n}\right)$ is a core of $A$, the assumption $D\left(A^{*}\right) \supset C_{0}^{\infty}\left(\mathbf{R}^{n}\right)$ in Proposition 2.3 follows from the rest of the assumptions. We also note that it suffices to assume $a_{\alpha} \in C^{|\alpha|}\left(\mathbf{R}^{n}\right)$.

For the use in $\S 4$, we briefly recall the theory of dispersive operators following Arendt-Chernoff-Kato [3], which gave final touches to the earlier results, e.g., Phillips [9], Sato [10], [11] and Hasegawa [4].

Let $E$ be a Banach lattice and let $\Phi$ be the canonical half norm on $E$ : $\Phi(u)=\left\|u^{+}\right\|(u \in E)$. Then a linear operator $A$ with domain $D(A)$ and range $R(A)$ in $E$ is said to be dispersive if $\Phi(u-\mu A u) \geq \Phi(u)$ holds for any $u \in D(A)$ and $\mu>0$. When $A$ further satisfies the condition $(I-\mu A) D(A)=E$ for any $\mu>0$ and is densely defined, it is called an $m$-dispersive operator. If a linear operator $A$ in $E$ has the property that $A-\lambda$ is dispersive [resp. $m$-dispersive] for some constant $\lambda, A$ is said to be quasi-dispersive [resp. quasi- $m$-dispersive]. By Theorem 2.4 in [3], a densely defined quasi-dispersive operator $A$ is closable and its closure $\tilde{A}$ is also quasi-dispersive. We call a densely defined linear operator $A$ essentially quasi- $m$-dispersive if its closure $\tilde{A}$ is quasi- $m$-dispersive.

Under these definitions, the following theorem is proved in [3] in a similar way as for the Lumer-Phillips theorem on contraction semigroups.

TheOREM A ([3] Theorem 4.1). A densely defined linear operator $A$ in a $B$ anach lattice $E$ is the generator of a $C_{0}$-semigroup of positive contractions if and only if $A$ is $m$-dispersive. Therefore if $A$ is essentially quasi-m-dispersive, then $\tilde{A}$ generates a positive $C_{0}$-semigroup.

The subdifferential $\partial \Phi$ of the canonical half norm $\Phi$ on $E$ at $u \in E$ is given by

$$
\partial \Phi(u)=\left\{f \in E^{*} ; f \geq 0,\|f\| \leq 1, f(u)=\left\|u^{+}\right\|\right\},
$$

which is nonvoid since $\Phi$ is continuous. Then a linear operator $A$ in $E$ is dispersive if and only if for any $u \in D(A)\langle A u, f\rangle \leq 0$ for some $f \in$ $\partial \Phi(u)$ ([3] Theorem 3.1). Therefore, in case $E=L^{p}\left(\mathbf{R}^{n}\right) A$ is dispersive if 
and only if

$$
\left\langle A u, F\left(u^{+}\right)\right\rangle \leq 0 \quad \text { for } u \in D(A),
$$

where

$$
F(u)=\|u\|^{2-p} u|u|^{p-2} \in L^{q}\left(\mathbf{R}^{n}\right) \quad(1 / p+1 / q=1)
$$

is the duality map, and $\langle$,$\rangle denotes the canonical duality between L^{p}$ and $L^{q}$. On the other hand, a linear operator $A$ in a real Banach space $X$ is called accretive if

$$
\|(A+\lambda) u\| \geq \lambda\|u\|
$$

for all $u \in D(A)$ and $\lambda>0$. $A$ is said to be $m$-accretive if it is accretive, densely defined, and the range of $A+\lambda$ is the whole space $X$ for some (equivalently for all) $\lambda>0$. It is well-known that $A$ is $m$-accretive if and only if $-A$ is the generator of a $C_{0}$-semigroup of contractions on $X([8] \mathrm{p}$. 14). Therefore if $A$ is an $m$-dispersive operator in a Banach lattice, then $-A$ is $m$-accretive.

Finally we note the following consequence of [3] Theorem 2.5 on addition of operators: Let $A, B$ be dispersive operators in a Banach lattice and suppose that $A$ is densely defined. Then the operator sum $A+B$ (with domain $D(A+B)=D(A) \cap D(B)$ and $(A+B) u:=A u$ $+B u$ for $u \in D(A+B)$ ) is also dispersive. Similarly the sum of two accretive operators in a Banach space is also accretive provided at least one of the two is densely defined.

3. Kato's inequality for differential operators. Let $\mathbf{N}$ be the set of all positive integers. In this section we shall use the multi-index notation: $\alpha=\left(\alpha_{1}, \ldots, \alpha_{n}\right)$ with $|\alpha|=\sum_{i=1}^{n} \alpha_{i}, \alpha_{i} \in \mathbf{N} \cup\{0\} ; D^{\alpha}=D_{1}^{\alpha_{1}} D_{2}^{\alpha_{2}} \cdots D_{n}^{\alpha_{n}}$, $D_{\imath}=\partial / \partial x_{i}(1 \leq i \leq n)$.

We consider the formal differential operator of order $m$

$$
\mathscr{A}=\sum_{|\alpha| \leq m} a_{\alpha}(x) D^{\alpha}
$$

where $a_{\alpha}(x)$ 's are assumed to be real valued functions in $C^{\infty}\left(\mathbf{R}^{n}\right)$.

Definition 3.1. A formal differential operator $\mathscr{A}$ of the form (3.1) is said to satisfy Kato's inequality if for any $u \in C_{0}^{\infty}\left(\mathbf{R}^{n}\right)$, the inequality

$$
\mathscr{A}|u| \geq(\operatorname{sgn} u) \mathscr{A} u
$$

holds in the sense of distribution, i.e., for any $\varphi \in C_{0}^{\infty}\left(\mathbf{R}^{n}\right)$ with $\varphi \geq 0$,

$$
\int \mathscr{A}^{*} \varphi(x)|u(x)| d x \geq \int \varphi(x)(\operatorname{sgn} u(x)) \mathscr{A} u(x) d x
$$

holds, where $\mathscr{A}^{*}$ denotes the formal adjoint of $\mathscr{A}$. 
In what follows we denote by $\mathscr{D}\left(\mathbf{R}^{n}\right)$ and $\mathscr{D}^{\prime}\left(\mathbf{R}^{n}\right)$ the space of all test functions (with the Schwartz topology) and distributions on $\mathbf{R}^{n}$, respectively, and consider that $\mathscr{D}\left(\mathbf{R}^{n}\right)$ is contained in $\mathscr{D}^{\prime}\left(\mathbf{R}^{n}\right)$.

First we prepare some facts about the change of variables of distributions. Although these facts are certainly known, we state them with proofs for later references. For the time being, we use two $\mathbf{R}^{n}$ 's, $\mathbf{R}_{x}^{n}$ and $\mathbf{R}_{y}^{n}$, and let $H: \mathbf{R}_{x}^{n} \rightarrow \mathbf{R}_{y}^{n}$ be a fixed non-singular linear mapping. Namely $y=$ $H(x):=C x\left(x \in \mathbf{R}_{x}^{n}\right)$, where $C=\left(c_{i j}\right)$ is an $n \times n$ matrix with real entries and $\operatorname{det} H=\operatorname{det} C \neq 0$. Then $H$ induces an isomorphism

$$
\varphi \in \mathscr{D}\left(\mathbf{R}_{y}^{n}\right) \mapsto \varphi \circ H \in \mathscr{D}\left(\mathbf{R}_{x}^{n}\right) .
$$

For $T \in \mathscr{D}^{\prime}\left(\mathbf{R}_{x}^{n}\right)$ define $\tilde{T} \in \mathscr{D}^{\prime}\left(\mathbf{R}_{y}^{n}\right)$ by

$$
\langle\tilde{T}, \varphi\rangle:=\langle T, \varphi \circ H\rangle \quad \text { for } \varphi \in \mathscr{D}\left(\mathbf{R}_{y}^{n}\right) .
$$

Under these notations we have the following

LeMMA 3.2. The mapping $T \mapsto \tilde{T}$ is a linear isomorphism from $\mathscr{D}^{\prime}\left(\mathbf{R}_{x}^{n}\right)$ to $\mathscr{D}^{\prime}\left(\mathbf{R}_{y}^{n}\right)$ satisfying

(a) $\left(\partial T / \partial x_{i}\right)^{\sim}=\sum_{j=1}^{n} c_{i i} \partial \tilde{T} / \partial y_{j}$,

(b) $(a T)^{\sim}=\left(a \circ H^{-1}\right) T$ for $a \in C^{\infty}\left(\mathbf{R}_{x}^{n}\right)$,

(c) $\tilde{T} \geq 0$ if and only if $T \geq 0$.

If in particular $T \in L_{\mathrm{loc}}^{1}\left(\mathbf{R}_{x}^{n}\right)$, then $\tilde{T} \in L_{\mathrm{loc}}^{1}\left(\mathbf{R}_{y}^{n}\right)$ and is given by $\tilde{T}=|\operatorname{det} H|^{-1}\left(T \circ H^{-1}\right)$.

Proof. It is obvious that the mapping $T \mapsto \tilde{T}$ becomes a linear isomorphism with property (c). Let $T \in \mathscr{D}^{\prime}\left(\mathbf{R}_{x}^{n}\right)$ and $\varphi \in \mathscr{D}\left(\mathbf{R}_{y}^{n}\right)$. Then we have

$$
\begin{aligned}
\left\langle\left(\partial T / \partial x_{i}\right)^{\sim}, \varphi\right\rangle & =\left\langle\partial T / \partial x_{i}, \varphi \circ H\right\rangle=-\left\langle T,\left(\partial / \partial x_{i}\right)(\varphi \circ H)\right\rangle \\
& =-\left\langle T,\left(\sum_{j=1}^{n} c_{j i} \frac{\partial \varphi}{\partial y_{j}}\right) \circ H\right\rangle=-\left\langle\tilde{T}, \sum_{j=1}^{n} c_{j i} \frac{\partial \varphi}{\partial y_{j}}\right\rangle \\
& =\left\langle\sum_{j=1}^{n} c_{j i} \frac{\partial \tilde{T}}{\partial y_{j}}, \varphi\right\rangle .
\end{aligned}
$$

This is nothing but (a). Furthermore, let $a \in C^{\infty}\left(\mathbf{R}_{x}^{n}\right)$ and $\varphi \in \mathscr{D}\left(\mathbf{R}_{y}^{n}\right)$. Then we obtain $(b)$ :

$$
\begin{aligned}
\left\langle(a T)^{\sim}, \varphi\right\rangle & =\langle a T, \varphi \circ H\rangle=\left\langle T,\left(\left(a \circ H^{-1}\right) \varphi\right) \circ H\right\rangle \\
& =\left\langle\tilde{T},\left(a \circ H^{-1}\right) \varphi\right\rangle=\left\langle\left(a \circ H^{-1}\right) \tilde{T}, \varphi\right\rangle .
\end{aligned}
$$

The last assertion is clear. 
The formal differential operator $\mathscr{A}$ of the form (3.1) is considered to be a linear operator from $\mathscr{D}^{\prime}\left(\mathbf{R}_{x}^{n}\right)$ into itself. The effect of the change of variables on $\mathscr{A}$ is given by the following

COROLlaRy 3.3. Let $\mathscr{A}$ be the formal differential operator of the form (3.1), and let $T \mapsto \tilde{T}$ be the mapping defined before Lemma 3.2. Then the linear operator

$$
\tilde{T} \in \mathscr{D}^{\prime}\left(\mathbf{R}_{y}^{n}\right) \mapsto(\mathscr{A} T)^{\sim} \in \mathscr{D}^{\prime}\left(\mathbf{R}_{y}^{n}\right) \quad\left(T \in \mathscr{D}^{\prime}\left(\mathbf{R}_{x}^{n}\right)\right)
$$

is given by the action of the following formal differential operator

$$
\tilde{\mathscr{A}}:=\sum_{|\alpha| \leq m}\left(a_{\alpha} \circ H^{-1}\right) \prod_{i=1}^{n}\left(\sum_{j=1}^{n} c_{j i} \frac{\partial}{\partial y_{j}}\right)^{\alpha_{i}},
$$

i.e., $(\mathscr{A} T)^{\sim}=\tilde{\mathscr{A}} \tilde{T}$ holds for $T \in \mathscr{D}^{\prime}\left(\mathbf{R}_{x}^{n}\right)$.

Proof. Repeated applications of Lemma 3.2. (a) and (b) prove the assertion.

\section{Moreover we have}

Proposition 3.4. Let $\mathscr{A}$ be the formal differential operator of the form (3.1) and let $\tilde{\mathscr{A}}$ be the one given by (3.4), which is obtained from $\mathscr{A}$ by the change of variables $y=H(x)=C x, C=\left(c_{i j}\right)(\operatorname{det} C \neq 0)$. Then $\tilde{\mathscr{A}}$ satisfies the Kato's inequality if and only if $\mathscr{A}$ does.

Proof. By using Lemma 3.2, we can show that for $u \in \mathscr{D}\left(\mathbf{R}_{x}^{n}\right)$

$$
\begin{aligned}
&(\mathscr{A}|u|-(\operatorname{sgn} u) \mathscr{A} u)^{-} \\
&=|\operatorname{det} H|^{-1}\left[\tilde{\mathscr{A}}\left|u \circ H^{-1}\right|-\left(\operatorname{sgn}\left(u \circ H^{-1}\right)\right) \tilde{\mathscr{A}}\left(u \circ H^{-1}\right)\right] .
\end{aligned}
$$

Hence the assertion follows from Lemma 3.2(c).

The main theorem in this section is stated as follows.

THEOREM 3.5. Let $a_{\alpha}$ be a real-valued function in $C^{\infty}\left(\mathbf{R}^{n}\right)$ for every $\alpha$ with $|\alpha| \leq m$. If the formal differential operator $\mathscr{A}=\sum_{|\alpha| \leq m} a_{\alpha}(x) D^{\alpha}$ satisfies Kato's inequality, then the order $m$ is at most 2. Furthermore, $\mathscr{A}_{1}:=\sum_{|\alpha|=2} a_{\alpha}(x) D^{\alpha}$ must be elliptic (including degenerate cases).

Proof. It suffices by translation to show that $a_{\alpha}(0)=0$ for any $\alpha$ with $|\alpha| \geq 3$. Set $k:=\max \left\{|\alpha| ; a_{\alpha}(0) \neq 0\right\}$. We shall show that Kato's inequality cannot be satisfied by $\mathscr{A}$ if $k \geq 3$. 
Step (1). First we consider a special case. Namely, suppose that

$$
a_{\alpha_{0}}(0) \neq 0 \text { for } \alpha_{0}=(k, 0, \ldots, 0) \text { with } k \geq 3 \text {. }
$$

Now let $u_{i}$ be a function in $\mathscr{D}(\mathbf{R})(i=1,2)$ and suppose that $u_{1}(t)=t$ near the origin and changes the sign only once, and that $u_{2}(t)=1$ near the origin and $u_{2} \geq 0$. Setting

$$
u(x):=u_{1}\left(x_{1}\right) \prod_{i=2}^{n} u_{2}\left(x_{i}\right)
$$

we see that $u \in \mathscr{D}\left(\mathbf{R}^{n}\right)$ and

$$
(\mathscr{A} u)(x)=\sum_{|\alpha| \leq m} a_{\alpha}(x) D_{1}^{\alpha_{1}} u_{1}\left(x_{1}\right) \prod_{i=2}^{n} D_{i}^{\alpha_{t}} u_{2}\left(x_{i}\right) .
$$

Next we calculate $(\mathscr{A}|u|)(x)$. The distributional derivative of $|u|$ can be written as

$$
\left(D^{\alpha}|u|\right)(x)=\left(D_{1}^{\alpha_{1}}\left|u_{1}\left(x_{1}\right)\right|\right) \prod_{i=2}^{n} D_{i}^{\alpha_{i}} u_{2}\left(x_{i}\right) .
$$

Note that the right-hand side should be regarded as a tensor product of distributions (see Schwartz [14], Chapter 4, §4, Theorem 7). In more detail,

$$
D_{1}^{\alpha_{1}}\left|u_{1}\left(x_{1}\right)\right|= \begin{cases}\left(\operatorname{sgn} u_{1}\left(x_{1}\right)\right) D_{1}^{\alpha_{1}} u_{1}\left(x_{1}\right) & \left(\alpha_{1}=0,1\right) \\ 2 \delta_{x_{1}=0}^{\left(\alpha_{1}-2\right)}+\left(\operatorname{sgn} u_{1}\left(x_{1}\right)\right) D_{1}^{\alpha_{1}} u_{1}\left(x_{1}\right) & \left(\alpha_{1} \geq 2\right),\end{cases}
$$

where $\delta_{x_{1}=0}^{\left(\alpha_{1}-2\right)}$ is the $\left(\alpha_{1}-2\right)$ th derivative of Dirac distribution. Thus we can write down $\mathscr{A}|u|$ as

$$
\begin{aligned}
(\mathscr{A}|u|)(x)= & 2 \sum_{\substack{\alpha_{1} \geq 2 \\
|\alpha| \leq m}} a_{\alpha}(x) \delta_{x_{1}=0}^{\left(\alpha_{1}-2\right)} \prod_{i=2}^{n} D_{i}^{\alpha_{t}} u_{2}\left(x_{i}\right) \\
& +\left(\operatorname{sgn} u_{1}\left(x_{1}\right)\right) \sum_{|\alpha| \leq m} a_{\alpha}(x) D_{1}^{\alpha_{1}} u_{1}\left(x_{1}\right) \prod_{i=2}^{n} D_{i}^{\alpha_{t}} u_{2}\left(x_{i}\right) .
\end{aligned}
$$

Therefore it follows from (3.6) that

$$
(\mathscr{A}|u|-(\operatorname{sgn} u) \mathscr{A} u)(x)=2 \sum_{\alpha_{1} \geq 2} a_{\alpha}(x) \delta_{x_{1}=0}^{\left(\alpha_{1}-2\right)} \prod_{i=2}^{n} D_{i}^{\alpha_{i}} u_{2}\left(x_{i}\right) .
$$

It remains to show that the right-hand side of (3.7) is not positive as a distribution. But, this is intuitively obvious. So, we give a proof for only one of four cases: let $k>2$ be even and $a_{\alpha_{0}}(0)<0$, where $\alpha_{0}$ is as in 
(3.5). Let $\psi_{i}$ be a function in $\mathscr{D}(\mathbf{R})(i=1,2)$ and suppose that $\psi_{1}(t)=t^{k-2}$ near the origin and $\psi_{1} \geq 0$. Next choose $b>0$ in such a way that

$$
[-b, b] \subset\left\{t \in \mathbf{R} ; u_{2}(t)=1\right\}
$$

and suppose that $\psi_{2}(0)=1, \psi_{2} \geq 0$ and supp $\psi_{2} \subset[-b, b]$. Setting

$$
\psi(x):=\psi_{1}\left(x_{1}\right) \prod_{i=2}^{n} \psi_{2}\left(x_{i}\right)
$$

we see that $\psi \in \mathscr{D}\left(\mathbf{R}^{n}\right)$. For a multi-index $\alpha$ with $\alpha_{1} \geq 2$, we have

$$
\begin{aligned}
\left\langle a_{\alpha}(x)\right. & \left.\delta_{x_{1}=0}^{\left(\alpha_{1}-2\right)} \prod_{i=2}^{n} D_{i}^{\alpha_{i}} u_{2}\left(x_{i}\right), \psi(x)\right\rangle \\
= & (-1)^{\alpha_{1}} \sum_{l=0}^{\left(\alpha_{1}-2\right)}\left(\begin{array}{c}
\alpha_{1}-2 \\
l
\end{array}\right) \int \cdots \int\left(D_{1}^{l} \psi\right)\left(0, x_{2}, \ldots, x_{n}\right) \\
& \times D_{1}^{\alpha_{1}-2-l} a_{\alpha}\left(0, x_{2}, \ldots, x_{n}\right) \prod_{i=2}^{n} D_{i}^{\alpha_{i}} u_{2}\left(x_{i}\right) d x_{2} \cdots d x_{n} .
\end{aligned}
$$

If $\alpha_{i}>0$ for some $i \geq 2$, then $D_{i}^{\alpha_{i}} u_{2}\left(x_{i}\right)=0$ on $[-b, b]$ for such $i$ and hence the integral on the right-hand side of (3.8) vanishes. This observation implies that $\langle\mathscr{A}|u|-(\operatorname{sgn} u) \mathscr{A} u, \psi\rangle / 2$ is equal to

$$
\begin{aligned}
\sum_{\alpha_{1} \geq 2}(-1)^{\alpha_{1}} \sum_{l=0}^{\alpha_{1}-2}\left(\begin{array}{c}
\alpha_{1}-2 \\
l
\end{array}\right) \int \cdots \int \psi_{1}^{(l)}(0) \prod_{i=2}^{n} \psi_{2}\left(x_{i}\right) \\
\quad \times\left(D_{1}^{\alpha_{1}-2-l} a_{\left(\alpha_{1}, 0, \ldots, 0\right)}\right)\left(0, x_{2}, \ldots, x_{n}\right) d x_{2} \cdots d x_{n} .
\end{aligned}
$$

Since $\psi_{1}^{(l)}(0)=0(0 \leq l \leq k-3)$ and $\psi_{1}^{(k-2)} \equiv(k-2)$ !, this can be simplified as

$$
(k-2) ! \int \cdots \int \prod_{i=2}^{n} \psi_{2}\left(x_{i}\right) a_{\alpha_{0}}\left(0, x_{2}, \ldots, x_{n}\right) d x_{2} \cdots d x_{n} .
$$

Taking a sufficiently small $b>0$, we see that the above integral can not be positive since $a_{\alpha_{0}}(0)<0$.

Step (2). Let $k$ be the number defined in the first paragraph of the proof and suppose that $a_{\alpha_{0}}(0)=0$ for $\alpha_{0}=(k, 0, \ldots, 0)$ with $k \geq 3$. By a linear transformation $y=H(x):=C x\left(x \in \mathbf{R}^{n}\right), \mathscr{A}$ is transformed into $\tilde{\mathscr{A}}$ in (3.4) (Corollary 3.3). The coefficient of $\left(\partial / \partial y_{1}\right)^{k}$ in $\tilde{\mathscr{A}}$ is given by

$$
\sum_{|\alpha|=k}\left(a_{\alpha} \circ H^{-1}\right) \prod_{i=1}^{n} c_{1 i}^{\alpha_{i}}
$$


and it does not vanish if we choose the non-singular matrix $C$ suitably. So, we see from Step (1) and Proposition 3.4 that $\mathscr{A}$ does not satisfy Kato's inequality if $k \geq 3$.

Step (3). Suppose that $\mathscr{A}$ satisfies Kato's inequality. Then, as was shown above, the principal part of $\mathscr{A}$ is given by

$$
\mathscr{A}_{1}:=\sum_{|\alpha|=2} a_{\alpha}(x) D^{\alpha} \text {. }
$$

Since it is known that the first order terms of $\mathscr{A}$ satisfies Kato's equality, i.e., $\left(\partial / \partial x_{i}\right)|u|=(\operatorname{sgn} u) \partial u / \partial x_{i}$ as a distribution $\left(u \in \mathscr{D}\left(\mathbf{R}^{n}\right)\right)$, we see that $\mathscr{A}_{1}$ also satisfies Kato's inequality. We shall show that $\mathscr{A}_{1}$ is elliptic at $x=0$.

First note that we can write

$$
\mathscr{A}_{1}=\sum_{i, j=1}^{n} a_{l j}(x) D_{i} D_{j}
$$

with $a_{i j}(x)=a_{j i}(x)$. So by an orthogonal transformation, $\mathscr{A}_{1}$ at $x=0$ is transformed into

$$
\sum_{j=1}^{n} a_{j}\left(\frac{\partial}{\partial y_{j}}\right)^{2}, \quad a_{j} \in \mathbf{R}(1 \leq j \leq n) .
$$

Then we can show that $a_{j} \geq 0$ for $1 \leq j \leq n$ by using Proposition 3.4 and applying a similar argument as in Step (1) (equation (3.7) for transformed $\mathscr{A}_{1}$ is again useful). Thus we are done.

By combining Proposition 2.3, Remark 2.4 and Theorem 3.5, we obtain the following

THEOREM 3.6. Let $\left\{T_{t}\right\}_{t \geq 0}$ be a positive $C_{0}$-semigroup on $L^{p}\left(\mathbf{R}^{n}\right)$ $(1 \leq p<\infty, n \in \mathbf{N})$ with generator $A$. Suppose that $C_{0}^{\infty}\left(\mathbf{R}^{n}\right)$ is a core of $A$ and $A$ is given as a differential operator on $C_{0}^{\infty}\left(\mathbf{R}^{n}\right)$ :

$$
A u(x)=\sum_{|\alpha| \leq m} a_{\alpha}(x) D^{\alpha} u(x) \quad\left(u \in C_{0}^{\infty}\left(\mathbf{R}^{n}\right) ; m \in \mathbf{N}\right),
$$

where $a_{\alpha}(x)$ 's are real-valued functions in $C^{\infty}\left(\mathbf{R}^{n}\right)$. Then the order of $A$ is at most 2, and the principal part of $A$ is elliptic including degenerate cases.

REMARK 3.7. A corresponding result for general domain $\Omega \subset \mathbf{R}^{n}$ will appear elsewhere. ${ }^{1}$

${ }^{1}$ Added in proof: R. Nagel et al. (eds.), Aspects of Positivity in Functional Analysis, North Holland Amsterdam, 1986. 
4. $m$-dispersiveness of second order degenerate elliptic differential operators on $\mathbf{R}^{n}$. This section is concerned with the quasi- $m$-dispersiveness (see \$2) of second order degenerate elliptic operators on $\mathbf{R}^{n}$. First let us consider the formal differential operator

$$
\mathscr{A} u:=-\sum_{j, k=1}^{n} D_{j}\left(a_{j k}(x) D_{k} u\right)+\sum_{j=1}^{n} a_{j}(x) D_{j} u+a_{0}(x) u,
$$

where $a_{j k}, a_{j}$ and $a_{0}$ are all real-valued functions on $\mathbf{R}^{n}$. Basic assumptions are stated as follows.

(I) $a_{j k} \in C^{2}\left(\mathbf{R}^{n}\right), a_{j} \in C^{1}\left(\mathbf{R}^{n}\right), a_{0} \in L^{\infty}\left(\mathbf{R}^{n}\right)$; the second order derivatives of $a_{j k}$ and the first order derivatives of $a_{j}$ are all bounded on $\mathbf{R}^{n}$.

(II) For any $x \in \mathbf{R}^{n}$ the matrix $\left(a_{j k}(x)\right)$ is positive semi-definite: for every $\xi \in \mathbf{R}^{n}$,

$$
\sum_{j, k=1}^{n} a_{j k}(x) \xi_{j} \xi_{k} \geq 0
$$

Let $A$ be the maximal operator in real $L^{p}=L^{p}\left(\mathbf{R}^{n}\right)(1<p<\infty)$ associated with $\mathscr{A}$ :

$$
\begin{aligned}
D(A) & :=\left\{u \in L^{p} ; \mathscr{A} u \in L^{p} \text { in the distribution sense }\right\}, \\
A u & :=\mathscr{A} u \text { for } u \in D(A) .
\end{aligned}
$$

Recently Baoswang Wong-Dzung [16] has proved the following

TheOREM B. Let $A$ be the operator as above. Then $-A$ is quasi-m-dispersive in $L^{p}(1<p<\infty)$ and $C_{0}^{\infty}\left(\mathbf{R}^{n}\right)$ is a core of $A$.

Now we consider the possibility to relax the condition $a_{0} \in L^{\infty}$. Let $V(x)>0$ be a function in $L_{\text {loc }}^{p}\left(\mathbf{R}^{n} \backslash\{0\}\right)$ and set

$$
V_{\varepsilon}(x):=V(x)[1+\varepsilon V(x)]^{-1}, \quad \varepsilon>0 .
$$

We denote by $B$ the maximal multiplication operator by $V(x)$ in $L^{p}$ :

$$
B u(x):=V(x) u(x) \text { for } u \in D(B):=\left\{u ; V(x) u \in L^{p}\right\} .
$$

Then $-B$ is $m$-dispersive in $L^{p}(1<p<\infty)$ and the bounded linear operator

$$
B_{\varepsilon} u(x):=V_{\varepsilon}(x) u(x), \quad u \in L^{p}, \varepsilon>0
$$

is related to the Yosida approximation of $-B$ (in the sense specified in §2) by the equation $B_{1 / \lambda}=-(-B)_{\lambda}(\lambda>0)$. Note that $B_{\varepsilon}$ is also written as $B_{\varepsilon}=B(1+\varepsilon B)^{-1}$. 
The purpose of this section is to prove the following

THEOREM 4.1. Let $A$ and $B$ be the operators in $L^{p}(1<p<\infty)$ as above. Assume that $V_{\varepsilon}$ belongs to $C^{1}\left(\mathbf{R}^{n}\right)$, and there exist nonnegative constants $c$, $a$ and $b\left(b \leq 4(p-1)^{-1}\right)$ such that for any $\varepsilon>0$ and $x \in \mathbf{R}^{n}$

$$
\begin{gathered}
\sum_{j, k=1}^{n} \frac{a_{j k}(x)}{V_{\varepsilon}(x)} D_{j} V_{\varepsilon} D_{k} V_{\varepsilon}+\frac{4}{p} \sum_{j=1}^{n} a_{j}(x) D_{j} V_{\varepsilon} \\
\leq c+a V_{\varepsilon}(x)+b\left[V_{\varepsilon}(x)\right]^{2} .
\end{gathered}
$$

In the case of $1<p<2$ assume further that $c=0$.

If $b<4(p-1)^{-1}$ then $-(A+B)$ is also quasi-m-dispersive in $L^{p}$. If $b=4(p-1)^{-1}$ then $-(A+B)$ is essentially quasi-m-dispersive on $D(A+B):=D(A) \cap D(B)$. In any case the closure of $-(A+B)$ is the generator of a positive $C_{0}$-semigroup on $L^{p}$.

The proof of this theorem is based on the following

LEMMA 4.2. Let $-A$ and $-B$ be $m$-dispersive operators in real $L^{p}\left(\mathbf{R}^{n}\right)$ $(1<p<\infty)$. Let $D$ be a core of $A$. Assume that there exist nonnegative constants $c$, $a$ and $b(b \leq 1)$ such that for any $u \in D$ and $\varepsilon>0$,

$$
\left\langle A u, F\left(B_{\varepsilon} u\right)\right\rangle \geq-c\|u\|^{2}-a\left\|B_{\varepsilon} u\right\|\|u\|-b\left\|B_{\varepsilon} u\right\|^{2}
$$

holds, where $B_{\varepsilon}=B(1+\varepsilon B)^{-1}$ and

$$
F\left(B_{\varepsilon} u\right)=\left\|B_{\varepsilon} u\right\|^{2-p} B_{\varepsilon} u \times\left|B_{\varepsilon} u\right|^{p-2} \in L^{q}=\left(L^{p}\right)^{*} \quad(1 / p+1 / q=1) .
$$

If $b<1$ then $-(A+B)$ with $D(A+B):=D(A) \cap D(B)$ is also $m$-dispersive in $L^{p}$. If $b=1$ then $-(A+B)$ is essentially $m$-dispersive on $D(A+B)$.

Proof. Since $A$ and $B$ are $m$-accretive in $L^{p}$ (see $\S 2$ ), it follows from Lemma 1.4 in Okazawa [7] that $A+B$ is $m$-accretive [resp. essentially $m$-accretive] if $b<1$ [resp. $b=1$ ]. Let $C$ denote $A+B$ or its closure according as $b<1$ or $b=1$. Since $-(A+B)$ is dispersive (see the last paragraph of §2) and the closure of dispersive operator is also dispersive ([3], Theorem 2.3), $-C$ is also dispersive. Thus we see that $-C$ is also $m$-dispersive in $L^{p}$.

Proof of Theorem 4.1. In order to apply Lemma 4.2, we shall show that for some constant $a^{*} \in \mathbf{R}$ and $b, c$ appearing in (4.1),

$$
4\left\langle A u, F\left(B_{\varepsilon} u\right)\right\rangle \geq-(p-1)\left(c\|u\|^{2}+a^{*}\left\|B_{\varepsilon} u\right\|\|u\|+b\left\|B_{\varepsilon} u\right\|^{2}\right)
$$


holds for any $u \in C_{0}^{\infty}\left(\mathbf{R}^{n}\right)$. Since

$$
\left|B_{\varepsilon} u(x)\right|^{p-2} B_{\varepsilon} u(x)=\left[V_{\varepsilon}(x)\right]^{p-1}|u(x)|^{p-2} u(x),
$$

we have

$$
\begin{aligned}
\langle A u,| & \left.\left.B_{\varepsilon} u(x)\right|^{p-2} B_{\varepsilon} u(x)\right\rangle \\
= & -\int_{\mathbf{R}^{n}} w(x)|u(x)|^{p-2} u(x) \sum_{j, k=1}^{n} D_{j}\left[a_{j k}(x) D_{k} u\right] d x \\
& +\int_{\mathbf{R}^{n}} w(x)|u(x)|^{p-2} u(x) \sum_{j=1}^{n} a_{j}(x) D_{j} u d x \\
& +\int_{\mathbf{R}^{n}} a_{0}(x) w(x)|u(x)|^{p} d x,
\end{aligned}
$$

where we set $w(x)=\left[V_{\varepsilon}(x)\right]^{p-1}$. Suppose $p \geq 2$. Then $w,|u|^{p-2} u \in$ $C^{1}\left(\mathbf{R}^{n}\right)$ and $D_{j}\left(|u|^{p-2} u\right)=(p-1)|u|^{p-2} D_{j} u, \quad D_{j}\left(|u|^{p}\right)=p|u|^{p-2} u D_{j} u$ hold $(1 \leq j \leq n)$. Therefore by the integration by parts it follows that

$$
\begin{aligned}
\left\langle A u,\left|B_{\varepsilon} u\right|^{p-2} B_{\varepsilon} u\right\rangle-\int_{\mathbf{R}^{n}} a_{0}(x) w(x)|u(x)|^{p} d x \\
=\int_{\mathbf{R}^{n}}|u|^{p-2} u \sum_{j, k=1}^{n} a_{j k}(x)\left(D_{j} w\right)\left(D_{k} u\right) d x \\
+(p-1) \int_{\mathbf{R}^{n}} w(x)|u|^{p-2} \sum_{j, k=1}^{n} a_{j k}(x)\left(D_{j} u\right)\left(D_{k} u\right) d x \\
+\frac{1}{p} \int_{\mathbf{R}^{n}} w(x) \sum_{j=1}^{n} a_{j}(x) D_{j}\left(|u|^{p}\right) d x .
\end{aligned}
$$

The sum of the first two terms on the right-hand side is not less than

$$
-[4(p-1)]^{-1} \int_{\mathbf{R}^{n}}[w(x)]^{-1}|u|^{p} \sum_{j, k=1}^{n} a_{j k} D_{j} w D_{k} w d x .
$$

Hence we obtain

$$
\begin{aligned}
\left\langle A u,\left|B_{\varepsilon} u\right|^{p-2} B_{\varepsilon} u\right\rangle \geq & -[4(p-1)]^{-1} \int_{\mathbf{R}^{n}} \frac{|u|^{p}}{w} \sum_{j, k=1}^{n} a_{j k}(x) D_{j} w D_{k} w d x \\
& -\frac{1}{p} \int_{\mathbf{R}^{n}}|u|^{p} \sum_{j=1}^{n} a_{j}(x) D_{j} w d x \\
& -\int_{\mathbf{R}^{n}} w(x)|u|^{p}\left[\frac{1}{p} \sum_{j=1}^{n} D_{j} a_{j}-a_{0}(x)\right] d x .
\end{aligned}
$$


This inequality holds even if $1<p<2$. In fact, by replacing $|u(x)|^{p-2}$ in $\left|B_{\varepsilon} u\right|^{p-2} B_{\varepsilon} u$ by $\left[|u(x)|^{2}+\delta\right]^{(p-2) / 2}(\delta>0)$ and letting $\delta \downarrow 0$ after the integration by parts, we obtain the above inequality in this case. By a straightforward calculation we see from (4.1) that

$$
\begin{gathered}
(p-1)^{-2} \sum_{j, k=1}^{n} \frac{a_{j k}(x)}{w(x)} D_{j} w D_{k} w+\frac{4}{p(p-1)} \sum_{j=1}^{n} a_{j}(x) D_{j} w \\
=\left[V_{\varepsilon}(x)\right]^{p-2}\left(\sum_{j, k=1}^{n} \frac{a_{j k}(x)}{V_{\varepsilon}(x)} D_{j} V_{\varepsilon} D_{k} V_{\varepsilon}+\frac{4}{p} \sum_{j=1}^{n} a_{j}(x) D_{J} V_{\varepsilon}\right) \\
\leq c\left[V_{\varepsilon}(x)\right]^{p-2}+a\left[V_{\varepsilon}(x)\right]^{p-1}+b\left[V_{\varepsilon}(x)\right]^{p},
\end{gathered}
$$

where $a$ is the constant appearing in (4.1).

Setting

$$
m=\sup \left\{p^{-1} \sum_{j=1}^{n} D_{j} a_{j}(x)-a_{0}(x) ; x \in \mathbf{R}^{n}\right\}, \quad a^{*}=a+4 m(p-1)^{-1}
$$

and using the Hölder inequality we obtain (4.2) for any $u \in C_{0}^{\infty}\left(\mathbf{R}^{n}\right)$. By Theorem $\mathrm{B}$, there exists a constant $M$ such that $-(A+M)$ is $m$-dispersive. For such an $M$, we set $a^{* *}=a^{*}+4 M(p-1)^{-1}$. Then we have

$$
\begin{aligned}
& \quad 4\left\langle(A+M) u, F\left(B_{\varepsilon} u\right)\right\rangle \\
& \quad \geq-(p-1)\left(c\|u\|^{2}+a^{* *}\|u\|\left\|B_{\varepsilon} u\right\|+b\left\|B_{\varepsilon} u\right\|^{2}\right)
\end{aligned}
$$

for any $u \in C_{0}^{\infty}\left(\mathbf{R}^{n}\right)$. Noting that $C_{0}^{\infty}\left(\mathbf{R}^{n}\right)$ is a core of $A+M$ (Theorem B), the conclusion follows from (4.3), Lemma 4.2 and Theorem A in $\S 2$.

Corollary 4.3. Let $A$ and $B$ be as in Theorem 4.1. Assume instead of (4.1) that $V(x)>0$ is of class $C^{1}\left(\mathbf{R}^{n}\right)$ and

$$
\sum_{j, k=1}^{n} \frac{a_{j k}(x)}{V(x)} D_{j} V D_{k} V+\frac{4}{p} \sum_{j=1}^{n} a_{j}(x) D_{J} V \leq b[V(x)+c]^{2},
$$

where $c$ and $b\left(b \leq 4(p-1)^{-1}\right)$ are nonnegative constants. Then the conclusion of Theorem 4.2 holds.

Proof. Set $W(x):=V(x)+c$ and $W_{\varepsilon}(x):=W(x)[1+\varepsilon W(x)]^{-1}$ for $\varepsilon>0$. Then by a simple calculation it can be shown that (4.4) implies (4.1) with $V_{\varepsilon}$ replaced by $W_{\varepsilon}$ and $a=c=0$, whereas with $b$ being the same as in (4.4). Therefore, by Theorem 4.1, $-(A+W)=-(A+V+c)$ is quasi$m$-dispersive or essentially quasi- $m$-dispersive on $D(A) \cap D(W)=D(A)$ $\cap D(V)$ according as $b<4(p-1)^{-1}$ or $b=4(p-1)^{-1}$, hence the corollary is proved. 
REMARK 4.4. The last paragraph of $\$ 2$ implies that the addition problem for $m$-dispersive operators is reduced to that of $m$-accretive operators. Hence there exists a possibility that the results on $m$-accretive operators contain the information about $m$-dispersive operators. In fact Okazawa [7] has implicitly shown that, for example, $\Delta-\exp \left(|x|^{k}\right)(k \geq 1)$ is essentially $m$-dispersive on $C_{0}^{\infty}\left(\mathbf{R}^{n}\right)$ in $L^{p}(1<p<\infty)$, where $\Delta$ means the Laplacian in $L^{p}$. For the details the reader is referred to [7].

\section{REFERENCES}

[1] W. Arendt, Generators of Positive Semigroups, in Infinite-Dimensional Systems, Lecture Notes in Math. No. 1076, Springer, 1984.

[2] __ Kato's Inequality: A Characterization of Generators of Positive Semigroups, Proceedings of the Royal Irish Academy, 84A (1984), 155-174.

[3] W. Arendt, P. Chernoff and T. Kato, A generalization of dissipativity and positive semigroups, J. Operator Theory, 8 (1982), 167-180.

[4] M. Hasegawa, On contraction semi-groups and (di)-operators, J. Math. Soc. Japan, 18 (1966), 290-302.

[5] T. Kato, Schrödinger operators with singular potentials, Israel J. Math., 13 (1972), 135-148.

[6] R. Nagel and H. Uhlig, An abstract Kato inequality for generators of positive operator semigroups on Banach lattices, J. Operator Theory, 6 (1981), 113-123.

[7] N. Okazawa, An $L^{p}$ theory for Schrödinger operators with nonnegative potentials, J. Math. Soc. Japan, 36 (1984), 675-688.

[8] A. Pazy, Semigroups of Linear Operators and Applications to Partial Differential Equations, Springer Verlag, New York, 1983.

[9] R. S. Phillips, Semigroups of positive contraction operators, Czechoslovak Math. J., (87) 12 (1962), 294-313.

[10] K. Sato, On the generators of non-negative contraction semigroups in Banach lattices, J. Math. Soc. Japan, 20 (1968), 423-436.

[11] _ On dispersive operators in Banach lattices, Pacific J. Math., 33 (1970), 429-443.

[12] H. H. Schaefer, Banach Lattices and Positive Operators, Grundlehren der mathematischen Wissenschaften, Band 215, Springer, Berlin-Heiderberg-New York, 1974.

[13] A. R. Schep, Weak Kato-inequalities and positive semigroups, Math. Z., 190 (1985), 305-314.

[14] L. Schwartz, Théorie des Distribution, Hermann, Paris, 1966.

[15] B. Simon, An abstract Kato's inequality for generators of positivity preserving semigroups, Indiana Univ. Math. J., 26 (1977), 1067-1073.

[16] B. Wong-Dzung, $L^{p}$-Theory of degenerate-elliptic and parabolic operators of second order, Proc. Royal Soc. Edinburgh, 95A (1983), 95-113.

[17] K. Yosida, Functional Analysis (6th edition), Grundlehren der mathematischen Wissenschaften, Band 123, Springer, Berlin-Heiderberg-New York, 1980.

Received October 2, 1984 and in revised form December 26, 1985. Supported by Grant-in-Aid for Scientific Research (No. 58540091), Ministry of Education.

FACULTY OF SCIENCE

SCIENCE UNIVERSITY OF TOKYO

WAKAMIYA-CHO 26, SHINJUKU-KU

TOKYO 162, JAPAN 



\section{PACIFIC JOURNAL OF MATHEMATICS EDITORS}

\author{
V. S. VARADARAJAN \\ (Managing Editor) \\ University of California \\ Los Angeles, CA 90024 \\ HERBERT ClEMENS \\ University of Utah \\ Salt Lake City, UT 84112 \\ R. FINN \\ Stanford University \\ Stanford, CA 94305
}

\author{
HERMANN FLASCHKA \\ University of Arizona \\ Tucson, AZ 85721 \\ RAMESH A. GANGOLLI \\ University of Washington \\ Seattle, WA 98195 \\ VAUghan F. R. JONES \\ University of California \\ Berkeley, CA 94720 \\ ROBION KIRBY \\ University of California \\ Berkeley, CA 94720
}

C. C. MOORE

University of California Berkeley, CA 94720

H. SAMELSON

Stanford University Stanford, CA 94305

HAROLD STARK

University of California, San Diego La Jolla, CA 92093

\section{ASSOCIATE EDITORS}
R. ARENS
E. F. BECKENBACH
B. H. NEUMANN
F. WOLF
K. YOSHIDA (1906-1982)

\section{SUPPORTING INSTITUTIONS}

UNIVERSITY OF ARIZONA

UNIVERSITY OF BRITISH COLUMBIA

CALIFORNIA INSTITUTE OF TECHNOLOGY

UNIVERSITY OF CALIFORNIA

MONTANA STATE UNIVERSITY

UNIVERSITY OF NEVADA, RENO

NEW MEXICO STATE UNIVERSITY

OREGON STATE UNIVERSITY
UNIVERSITY OF OREGON UNIVERSITY OF SOUTHERN CALIFORNIA

STANFORD UNIVERSITY

UNIVERSITY OF HAWAII

UNIVERSITY OF TOKYO

UNIVERSITY OF UTAH

WASHINGTON STATE UNIVERSITY

UNIVERSITY OF WASHINGTON 


\section{Pacific Journal of Mathematics}

Vol. 125, No. 1 September, 1986

Gilles Christol, Fonctions et éléments algébriques $\ldots \ldots \ldots \ldots \ldots \ldots \ldots \ldots$

Jo-Ann Deborah Cohen, Extensions of valuation and absolute valued

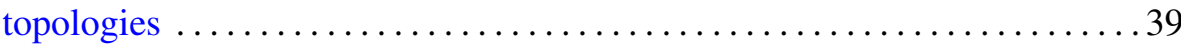

Miriam Cohen, Smash products, inner actions and quotient rings . . . . . . 45

Mikio Furushima, On the singular $K-3$ surfaces with hypersurface

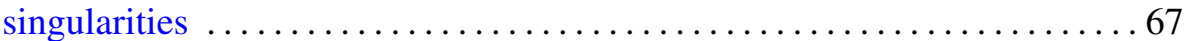

Gerhard Gierz and Boris Shekhtman, A duality principle for rational

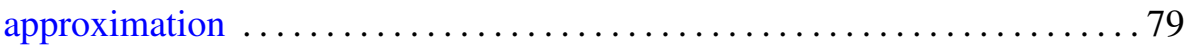

Anthony Wood Hager, A description of HSP-like classes, and

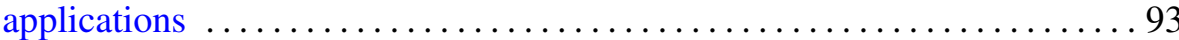

George Alan Jennings, Lines having high contact with a projective

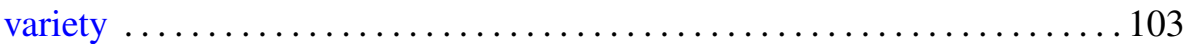

John Lott, Eigenvalue bounds for the Dirac operator . . . . . . . . . . . 117

Denis Laurent Luminet, A functional calculus for Banach PI-algebras . . . . 127

Shizuo Miyajima and Noboru Okazawa, Generators of positive

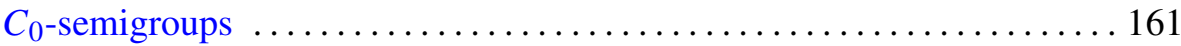

Takemi Mizokami, On functions and stratifiable $\mu$-spaces $\ldots \ldots \ldots \ldots \ldots 177$

Jeff Parker, 4-dimensional $G$-manifolds with 3-dimensional orbits . . . . . 187

Elias Saab and Paulette Saab, On Peł czyński's properties (V) and (V*) . . 205

Elmar Schrohe, The symbols of an algebra of pseudodifferential operators

Aart van Harten and Els Vader-Burger, Approximate Green functions as a tool to prove correctness of a formal approximation in a model of competing and diffusing species

Stephen Watson, Using prediction principles to construct ordered

continua 